\title{
Oscillations and Rotations of Elliptical Warm-Core Rings
}

\author{
Benoit CUShMan-Roisin \\ Mesoscale Air-Sea Interaction Group, Florida State University, Tallahassee
}

WolfGang H. HeIL

Department of Mathematics, Florida State University, Tallahassee

DORON NoF

Department of Oceanography, Florida State University, Tallahassee

\begin{abstract}
Exact analytical solutions are found to describe $f$ plane time-dependent, elliptical warm-core rings where the interface intersects the surface along the periphery. The space variables can be eliminated to reduce the problem to a system of differential equations in time. The motion of the center of mass is resolved and subtracted. Small departures from circular shape have three intrinsic frequencies: two are inertial and superinertial, while the third is a low-frequency mode that corresponds to a slow rotation of the elliptical eddy without change in shape. An exact solution for steadily rotating elliptical eddies of finite eccentricity (named Rodons) is also found and discussed. Comparison with elliptical warm-core Gulf Stream rings shows that this low-frequency mode may explain their clockwise rotation. The solution also shows the existence of a periodically reversing deformation field that, combined with a reasonable amount of mixing, would result in an efficient homogenization of the water contained in the ring. An exact solution that corresponds to the pulsation of a circular eddy has also been found.
\end{abstract}

\section{INTRODUCTION}

Warm-core rings shed by the Gulf Stream are now well documented [e.g., Csanady, 1979; Joyce, 1984; Joyce et al., 1984]. They are clockwise rotating, high-pressure centers bound underneath by a strong thermocline that rises around the warm-water pool and reaches the surface, forming a front along the ring's periphery. This edge is often elliptical [e.g., Brown et al., 1984]. Other warm-core rings in the world ocean (Loop Current rings and warm rings observed south of the Kuroshio) also display a tendency to have an elliptical edge [Kirwan et al.. 1984; Kitano, 1974]. Mied et al. [1983] described in detail an elongated cold-core ring. The ellipticity is probably a result of nonaxisymmetric birthing conditions and subsequent shear during the eddy's life [Nof, 1984] or inherent instability [Saunders, 1973; Griffiths and Linden, 1981; Mied et al., 1983]. The noted absence of elongated elliptical rings suggests that high eccentricities lead to instability and self destruction such that only quite circular rings can survive. The excellent satellite coverage of the slope water region northwest of the Gulf Stream that exists today [e.g., Evans et al., 1984] shows that a typical eccentricity is $80 \%$ and that such a typical warm-core ring rotates clockwise at a rate of about $9^{\circ}$ per day. For cold-core Gulf Stream rings, Spence and Legeckis [1981] report counterclockwise rotations of about $22^{\circ}$ per day.

So far, theoretical oceanographers have proposed very few models of warm-core rings surrounded by an interface that extends to the surface, forming a containing front. Calculations on an $f$ plane, based on the assumptions of uniform potential vorticity and circular shape, have been presented by Csanady [1979] and Flierl [1979]. Such circular rings are steady. Nof $[1981,1983]$ used an integration method to study

Copyright 1985 by the American Geophysical Union.

Paper number $5 \mathrm{C} 0244$.

0148-0227/85/005C-0244\$05.00 the westward drift induced by the beta effect without having to resolve the eddy's internal structure. His results were somewhat extended by Killworth [1983], who showed that the beta-effect correction to an otherwise $f$ plane circular eddy is a meridional shift that almost preserves the circular shape and that stability is ensured. Very recently, Nof [1985] discussed the ellipticity induced by the presence of a mean shear in the particular circumstance when a steady equilibrium is reached between shear and ellipticity.

Besides the available observations, there is no study discussing the time-dependent behavior of elliptical eddies. The reason probably resides in the fact that a surfacing interface precludes the use of any quasi-geostrophic model and severely complicates the use of numerical models. Ikeda [1981] presented a stability analysis of a two-layer Gaussian eddy but resorted to a quasi-geostrophic model. This limitation prevents applicability to intense structures such as Gulf Stream rings. The particular analytical solution presented here is proposed in order to remedy partly the present lack of theoretical support at a time when the increased satellite coverage encourages new theories.

By contrast, in the absence of rotation and stratification there exists an old exact analytical solution that represents the steady rotation of an elliptical patch of uniform vorticity. It is known as Kirchhoff's vortex [Kirchhoff, 1876; Hill, 1884; $L a m b, 1932]$. Its stability has been investigated by Love [1893], who stated that if the ratio of the major to the minor axes is more than three the vortex is unstable to infinitesimal perturbations. It would be very helpful if a corresponding result could be obtained for elliptical warm-core rings. Because of mathematical complications, such stability study has not been included in the present work.

In two classic papers, Ball $[1963,1965]$ studied the motion of a homogeneous fluid contained in an elliptic paraboloid. His aim was the investigation of oscillatory modes in shallow basins, as these modes can influence tidal motions in seas and 
lakes. Although the present problem is directed toward upperocean eddies, equations are identical (gravity becoming reduced gravity, the topography effect being set to zero), and some mathematical developments are similar. The two solutions of the complete nonlinear problem that are presented in section 4 are new and were not known to Ball.

The organization of the paper is as follows. Section 2 presents the method of solution, which by eliminating the spatial dependence, reduces the problem to a set of coupled nonlinear ordinary differential equations in time. The motion of the eddy's center is resolved and eliminated from the system. Then, equilibrium solutions are isolated, and numerical solutions of the nonlinear system of ordinary differential equations are presented. These solutions help identify the various modes of oscillation. Section 3 treats the behavior of small departures from circular shape. This study isolates the possible modes and shows that one mode among three has a low-frequency character that may correspond to the observed slow, clockwise rotation of warm-core rings. Based on the numerical results of section 2 and the linearized solution of section 3 , section 4 is devoted to two distinct completely nonlinear solutions, namely a uniformly rotating ellipse and a pulsating circle. Section 5 pursues the study of this mode by comparing analytical results and observations. Section 6 concludes the work and calls for a stability study of time-dependent elliptical eddies. An appendix discusses the invariant properties.

\section{Equations and Solution of Spatial Structure}

\section{2a. Removal of Spatial Dependence}

A reduced-gravity model (only upper layer of fluid in motion) is the simplest model that can allow interface surfacing. Despite its restricted vertical representation, it has the advantage of combining adequate horizontal description with simple equations. For instance, most stability studies of frontal systems are based on the reduced-gravity model [Stern, 1980; Stern et al., 1982; Griffiths et al., 1982; Paldor, 1983a, b], and the few models of warm-core rings mentioned in the introduction are also constructed with a reduced-gravity formalism. The governing equations on an $f$-plane are, after scaling,

$$
\begin{gathered}
u_{t}+u u_{x}+v u_{y}-v=-h_{x} \\
v_{t}+u v_{x}+v v_{y}+u=-h_{y} \\
h_{t}+(h u)_{x}+(h v)_{y}=0
\end{gathered}
$$

where the upper-layer depth $h$ has been scaled by a reference depth $H$ (say the maximum depth at some specified time); the velocity components $u$ and $v$ by the gravity wave speed $\left(g^{\prime} H\right)^{1 / 2}$, where $g^{\prime}$ is the reduced gravity; the coordinates $x$ and $y$ by the radius of deformation $\left(g^{\prime} H\right)^{1 / 2} / f$, where $f$ is the constant Coriolis parameter; and the time $t$ by the inertial time $1 / f$. It is noted that with this scaling all terms in the momentum and continuity equations are potentially of the same order of magnitude, and the motion is ageostrophic.

The eddy region is defined where $h$ is positive and its edge as the closed curve where $h(x, y, t)=0$. Namely, the focus is on lenslike eddies embedded in a resting ocean. A key to the method of solution is to realize that both the kinematic and dynamic boundary conditions to be imposed along the edge are always automatically satisfied. Indeed, the kinematic condition that stipulates that a particle on the edge remains on the edge at all times is readily met by $(1 c)$ : where $h=0,(1 c)$ becomes $h_{t}+u h_{x}+v h_{y}=0$, and those particles conserve their $h=0$ and remain on the edge. The dynamic condition re- quires continuity of pressure across the edge, i.e., $g^{\prime} h$ must be uniform, which of course is met where $h$ vanishes. (Note that since the model is inviscid the tangential velocity component is not necessarily continuous across the edge.) In summary, any solution that satisfies $(1 a-c)$ will automatically satisfy the boundary conditions and, hence, is an acceptable solution to the problem. (One regrets that this advantage is lost as two or more layers are in motion.)

One particular solution that obeys $(1 a-c)$ is where the depth is a quadratic function of space and the velocity components linear functions [Ball, 1965]:

$$
\begin{gathered}
h=A x^{2}+2 B x y+C y^{2}+2 D x+2 E y+F \\
u=U_{1} x+U_{2} y+U_{0} \\
v=V_{1} x+V_{2} y+V_{0}
\end{gathered}
$$

where all 12 coefficients are time dependent in general. The eddy is of finite size if the edge is a closed curve, i.e., if

$$
\Delta=A C-B^{2}>0
$$

in which case the eddy's rim is an ellipse (a circle if $A=C$ and $B=0$ ).

The implementation of $(2 a-c)$ in $(1 a-c)$ yields to the following 12 nonlinear ordinary differential equations, where an overdot represents a time derivative:

$$
\begin{aligned}
\dot{U}_{1} & =-U_{1}{ }^{2}-U_{2} V_{1}+V_{1}-2 A \\
\dot{V}_{1} & =-U_{1} V_{1}-V_{1} V_{2}-U_{1}-2 B \\
\dot{U}_{2} & =-U_{1} U_{2}-U_{2} V_{2}+V_{2}-2 B \\
\dot{V}_{2} & =-U_{2} V_{1}-V_{2}{ }^{2}-U_{2}-2 C \\
\dot{A} & =-\left(3 U_{1}+V_{2}\right) A-2 V_{1} B \\
\dot{B} & =-U_{2} A-2\left(U_{1}+V_{2}\right) B-V_{1} C \\
\dot{C} & =-2 U_{2} B-\left(U_{1}+3 V_{2}\right) C \\
\dot{U}_{0} & =-U_{0} U_{1}-U_{2} V_{0}+V_{0}-2 D \\
\dot{V}_{0} & =-U_{0} V_{1}-V_{0} V_{2}-U_{0}-2 E \\
\dot{D} & =-U_{0} A-V_{0} B-\left(2 U_{1}+V_{2}\right) D-V_{1} E \\
\dot{E} & =-U_{0} B-V_{0} C-U_{2} D-\left(U_{1}+2 V_{2}\right) E \\
\dot{F} & =-2 U_{0} D-2 V_{0} E-\left(U_{1}+V_{2}\right) F
\end{aligned}
$$

At this stage the spatial structure of the eddy has been solved, and only the time dependence of the problem remains. The most general solution to the above set of equations has not been found, and the focus is placed on several particular solutions. The present paper presents the equilibrium solutions (subsection $2 c$ ), the solutions corresponding to small elliptical departures from a circular eddy (section 3), two distinct exact nonlinear solutions (section 4), as well as some numerical solutions (subsection $2 d$ ).

\section{2b. General Properties and Motion of Center of Mass}

Among these 12 equations, the first seven make a subset of equations for $U_{1}, V_{1}, U_{2}, V_{2}, A, B$, and $C$ that can be solved first. Once these variables are known, the next four equations can be solved for $U_{0}, V_{0}, D$, and $E$, and when these variables are known, the last equation will provide $F$. From (8), (9), and (10) one can see that

$$
\Delta=-4\left(U_{1}+V_{2}\right) \Delta
$$




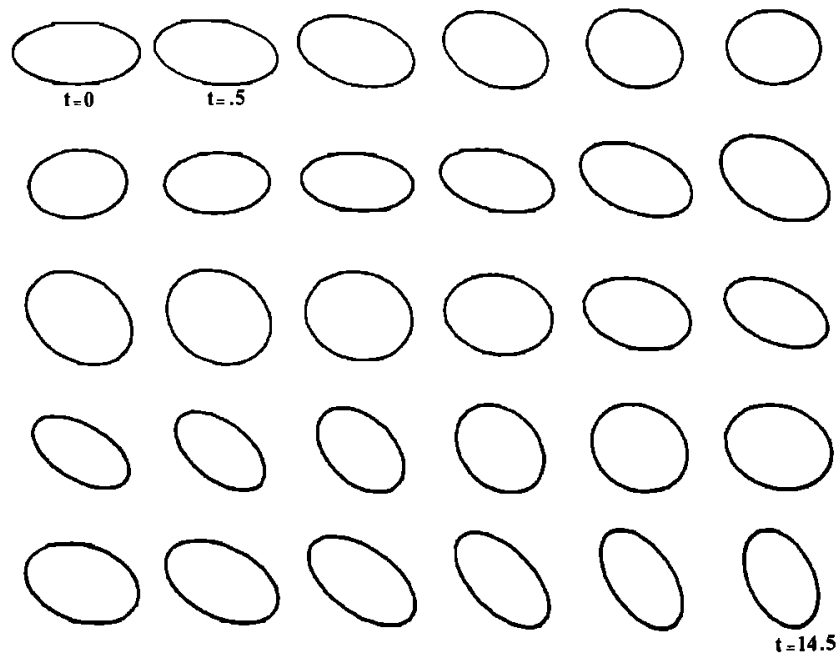

Fig. 1. Time sequence of the eddy rim at constant time intervals ( $2 \pi$ corresponds to one inertial period). Note the tendency of clockwise rotation, superimposed upon which are shape oscillations between ellipse and circle and pulsations between expansion and contraction.

delta never changes sign, and the eddy remains elliptical at all times.

The eddy's center is the point where the depth gradient vanishes; it has the coordinates

$$
x_{0}=\frac{B E-D C}{\Delta} \quad y_{0}=\frac{B D-A E}{\Delta}
$$

The time rate of change of these coordinates that gives the velocity of the center point yields, from (4) to (14),

$$
\dot{x}_{0}=U_{1} x_{0}+U_{2} y_{0}+U_{0} \quad \dot{y}_{0}=V_{1} x_{0}+V_{2} y_{0}+V_{0}
$$

One notes that this center-point velocity is nothing else but the actual particle velocity and thus that, for the present particular spatial structure, the center point is a material point. Since the pressure force at the center is always zero, its trajectory is an inertial circle. To verify this mathematically, one computes the acceleration and finds

$$
\ddot{x}_{0}=\dot{y}_{0} \quad \ddot{y}_{0}=-\dot{x}_{0}
$$

which shows that the trajectory of the center point is a clockwise circle

$$
x_{0}=X_{0}+R \sin (t+\phi) \quad y_{0}=Y_{0}+R \cos (t+\phi)
$$

where $X_{0}, Y_{0}, R$, and $\phi$ are to be evaluated from the initial conditions, the inertial-circle radius $R$ being equal to the magnitude of the initial velocity of the center point.

Now that the motion of the center is known, one will heretofore study the rotation and deformation of the eddy around that point by introducing the change of variables

$$
\begin{gathered}
x^{\prime}=x-x_{0}(t) \quad y^{\prime}=y-y_{0}(t) \quad t^{\prime}=t \\
u^{\prime}=u-\dot{x}_{0}(t) \quad v^{\prime}=v-\dot{y}_{0}(t)
\end{gathered}
$$

The implementation of these new coordinates shows that the first seven coupled equations (4)-(10) remain unchanged, the next four equations $(11)-(14)$ reduce to an identity, and the last equation (15) becomes

$$
\dot{H}_{0}=-\left(U_{1}+V_{2}\right) H_{0}
$$

where $H_{0}$ is a new quantity defined by

$$
H_{0}=\frac{1}{\Delta}\left|\begin{array}{lll}
A & B & D \\
B & C & E \\
D & E & F
\end{array}\right|
$$

and is such that $h=A x^{\prime 2}+2 B x^{\prime} y^{\prime}+C y^{\prime 2}+H_{0}$. In other words, $H_{0}$ is the maximum depth at the center $\left(H_{0}>0\right)$. With this change of variables and in the rest of the paper, the center of mass is a fixed point. This elimination simplifies the algebra, while no physical process has been filtered out.

The inviscid model $(1 a-c)$ conserves volume, angular momentum, potential vorticity, and energy. The corresponding invariant quantities are presented in the appendix.

\section{2c. Equilibrium Solutions}

With the aid of these prerequisites, one now turns to the steady solutions of system (4) to (10) and (16). For a timeindependent solution the coordinate axes can always be chosen so that they correspond to the principal directions of the ellipse $(B=0)$, and the maximum depth at center can be used as the scale for the interfacial depth $\left(H_{0}=1\right)$. After setting all time derivatives to zero, calculations show that only two equilibrium solutions exist. One is the circular ring

$$
h=1-\frac{1}{2} W(1-W)\left(x^{2}+y^{2}\right) \quad u=W y \quad v=-W x
$$

where $W$ is an arbitrary constant related to both the swirl speed and the ring's radius. This constant must range between 0 and 0.5 , otherwise either the interface lies above the surface or the ring is inertially unstable (negative potential vorticity). Small values of $W$ correspond to wide eddies. The other solution corresponds to an infinite band of light water (oriented in any direction but expressed here as it extends in the $y$ direction):

$$
h=1-x^{2} / L^{2} \quad u=0 \quad v=-2 x / L^{2}
$$

where $L$ is half its width. It is worth noting that these two equilibrium states are the two extreme elliptical configurations.

\section{2d. Numerical Solutions}

Before isolating some particular analytical solutions in the next two sections, it is interesting to present some numerical solutions of the complete nonlinear system. These will shed some light of the various possible behaviors that an elliptical eddy can exhibit. Figure 1 shows the time evolution of the eddy's elliptical edge at regular time intervals. The initial conditions correspond to the circular solution (17) with $W=1 / 3$, on which is superimposed a degree of ellipticity. The evolution shows a tendency of the eddy to rotate clockwise with two added oscillations. The shape oscillates between an ellipse of some maximum eccentricity and a circle, and the area pulsates as the eddy successively expands and contracts. The evolution time scale is clearly on the order of the inertial period ( $2 \pi$ for the present time scaling).

McCalpin [1984] performed numerical experiments on cyclonic, cold-core rings with Gaussian radial structure. After decomposition into azimuthal modes, his results showed that these modes are rotating counterclockwise (as can be expected in a cyclonic context where the swirl motion is also counterclockwise) and that there is a definite trend toward energy leaving the higher azimuthal modes to return to the basic, circular state. In particular an elliptical eddy shows the tendency of becoming irrevocably circular. Numerical experiments 


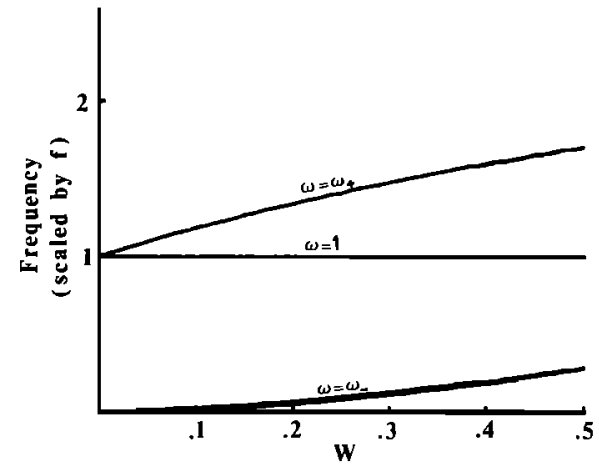

Fig. 2. Plot of the various frequencies for small elliptical departures from a circular eddy versus the basic-state parameter $W$. Small $W$ values correspond to wide eddies, while $W=1 / 2$ corresponds to the smallest realizable eddy. Three frequencies are nonzero, and one is zero.

by Ikeda [1981] also suggest the same behavior. This trend is more pronounced as the Rossby number increases. This major difference between the Ikeda-McCalpin results on one side and the present integration on the other side may be attributable to edge effects. Indeed, in a Gaussian-type eddy the slowly rotating outer portion may act as a damper on the crests of azimuthal heterogeneities. This is only a conjecture, but were it proven to be correct, the conclusion would be that edge effects, absent from the present analysis, can be quite important.

\section{Small Departures From Circular Shape}

The last section showed that, after the elimination of spatial dependence, the problem is reduced to a set of coupled, nonlinear, ordinary differential equations $((14)-(15))$ that cannot be solved analytically in their generality. Besides the numerical time integration of the preceding section, another approach remains linearization about a known simple solution. This section studies the behavior of small elliptical departures from the known steady, circular solution (17). This equilibrium state is quite close to the structure of actual rings. Observations made during the 1960's and 1970's suggest that the maximum swirl speed occurs near the edge [see Csanady, 1979, p. 779], as is the case of (17). More recent observations [Joyce, 1984] suggest that the ring contains a core with a linearly distributed orbital velocity and an outer layer with a decaying speed. Namely, it appears that, although the velocity peaks at some distance from the edge, most of the fluid contained in the ring rotates in a solid body rotation as stated by (17).

Noting by lowercase letters the infinitesimal perturbations from the equilibrium circular solution (17) $[A=C=-W(1$ $\left.-W) / 2, U_{2}=-V_{1}=W, H_{0}=1, B=U_{1}=V_{2}=0\right]$, one obtains after linearization

$$
\begin{gathered}
\dot{u}_{1}=(1-W) v_{1}+W u_{2}-2 a \\
\dot{v}_{1}=-(1-W) u_{1}+W v_{2}-2 b \\
\dot{u}_{2}=-W u_{1}+(1-W) v_{2}-2 b \\
\dot{v}_{2}=-W v_{1}-(1-W) u_{2}-2 c \\
\dot{a}=\frac{1}{2} W(1-W)\left(3 u_{1}+v_{2}\right)+2 W b \\
\dot{b}=\frac{1}{2} W(1-W)\left(v_{1}+u_{2}\right)-W a+W c \\
\dot{c}=\frac{1}{2} W(1-W)\left(u_{1}+3 v_{2}\right)-2 W b \\
h_{0}=-u_{1}-v_{2}
\end{gathered}
$$

This $8 \times 8$ linear system will yield four uncoupled modes of oscillation or growth/decay. Forming the following combinations, (19) $+(22),(20)-(21)$, and $(23)+(25)$, one obtains a $3 \times 3$ subset

$$
\begin{aligned}
\left(u_{1}+v_{2}\right)^{\prime} & =(1-2 W)\left(v_{1}-u_{2}\right)-2(a+c) \\
\left(v_{1}-u_{2}\right)^{\prime} & =-(1-2 W)\left(u_{1}+v_{2}\right) \\
(a+c)^{-} & =2 W(1-W)\left(u_{1}+v_{2}\right)
\end{aligned}
$$

where $u_{1}+v_{2}$ and $v_{1}-u_{2}$ are the divergence and vorticity perturbations, respectively, and $a+c$ can be thought of as the perturbation to the total Gaussian curvature of the depth field. The most general solution is

$$
\begin{aligned}
u_{1}+v_{2} & =2 N \cos (t+\phi) \\
v_{1}-u_{2} & =-2(1-2 W) N \sin (t+\phi)+2 M \\
a+c & =4 W(1-W) N \sin (t+\phi)+(1-2 W) M
\end{aligned}
$$

The coefficients $M, N$, and $\phi$ are three arbitrary constants of integration. The solution is oscillatory and thus stable. The frequency of oscillation is unity (inertial), while the additive constant corresponds to a zero-frequency mode.

Two frequencies have been isolated. The remaining two frequencies of the system (19)-(26) are then obtained by forming the following combinations

$$
\begin{aligned}
\left(u_{1}-v_{2}\right) & =\left(v_{1}+u_{2}\right)-2(a-c) \\
\left(v_{1}+u_{2}\right) & =-\left(u_{1}-v_{2}\right)-4 b \\
(a-c) & =W(1-W)\left(u_{1}-v_{2}\right)+4 W b \\
2 b & =W(1-W)\left(v_{1}+u_{2}\right)-2 W(a-c)
\end{aligned}
$$

which form a $4 \times 4$ system. The variables are now $u_{1}-v_{2}$ and $v_{1}+u_{2}$, the perturbations of the normal and shear deformation rates, respectively, and $a-c$ and $b$, two complementary measures of the degree of ellipticity. A solution of the type $e^{i \omega t}$ is possible, provided that

$$
\omega^{4}-(1+4 W) \omega^{2}+4 W^{4}=0
$$

i.e., for frequencies equal to

$$
\omega=\frac{1}{2}\left[(1+2 W) \pm\left(1+4 W-4 W^{2}\right)^{1 / 2}\right]
$$

The frequencies are all real, and these two modes, too, are stable. A plot of the three nonzero modal frequencies is presented on Figure 2. From this figure it is evident that one frequency is subinertial (minus sign in brackets of (28)) and one frequency is superinertial (plus sign in brackets of (28)). But what is more important to note is that in the limit of a wide eddy compared to the radius of deformation (small $W$ ), the subinertial frequency is very low. The asymptotic expression obtained from (28),

$$
\omega \approx 2 W^{2}
$$

increases less than linearly with $W$. For a circular eddy of radius equal to 4 times the radius of deformation, $W=0.15$, $\omega=0.034$, and the period of oscillation is 21 days.

Now that all frequencies have been isolated, the most general solution to the linearized problem can be constructed. The time-dependent coefficients are displayed at top of next page, where the basic state and all four modes are made explicit. The solution is then obtained by reintroducing the spatial dependence $\left(u=U_{1} x+U_{2} y, v=V_{1} x+V_{2} y, h=A x^{2}+2 B x y\right.$ $\left.+C y^{2}+H_{0}\right)$. The constants $M, M^{\prime}, N, P$, and $Q$ are arbitrary 
$\left.\left[\begin{array}{c}U_{1} \\ V_{1} \\ U_{2} \\ V_{2} \\ A \\ B \\ C \\ H_{0}\end{array}\right]=\left[\begin{array}{c}0 \\ -W \\ W \\ 0 \\ -\frac{1}{2} W(1-W) \\ 0 \\ -\frac{1}{2} W(1-W) \\ 1\end{array}\right]+M\left[\begin{array}{c}0 \\ 1 \\ -1 \\ 0 \\ \frac{1}{2}(1-2 W) \\ 0 \\ \frac{1}{2}(1-2 W) \\ M^{\prime}\end{array}\right]+N\left[\begin{array}{c}\cos (\tau+\phi) \\ (2 W-1) \sin (\tau+\phi) \\ (1-2 W) \sin (\tau+\phi) \\ \cos (\tau+\phi) \\ 2 W(1-W) \sin (\tau+\phi) \\ 0 \\ 2 W(1-W) \sin (\tau+\phi) \\ -2 \sin (\tau+\phi)\end{array}\right]+P\left[\begin{array}{c}\cos \omega_{-}(\tau+\chi) \\ -\sin \omega_{-}(\tau+\chi) \\ -\sin \omega_{-}(\tau+\chi) \\ -\cos \omega_{-}(\tau+\chi) \\ \alpha_{-} \sin \omega_{-}(\tau+\chi) \\ \alpha_{-} \cos \omega_{-}(\tau+\chi) \\ -\alpha_{-} \sin \omega_{-}(\tau+\chi) \\ 0\end{array}\right] \begin{array}{c}\cos \omega_{+}(\tau+\psi) \\ -\sin \omega_{+}(\tau+\phi) \\ -\sin \omega_{+}(\tau+\phi) \\ -\cos \omega_{+}(\tau+\psi) \\ \alpha_{+} \sin \omega_{+}(\tau+\psi) \\ \alpha_{+} \cos \omega_{+}(\tau+\psi) \\ -\alpha_{+} \sin \omega_{+}(\tau+\psi) \\ 0\end{array}\right]$

(small) amplitudes, while $\phi, \chi$, and $\psi$ are arbitrary phases. The constants $\alpha_{ \pm}$are defined by

$$
\alpha=\frac{1}{4}\left[2 W-1 \pm\left(1+4 W-4 W^{2}\right)^{1 / 2}\right]
$$

The "mode" of amplitude $M$ is a steady state that corresponds to a small change to the parameter $W$ of the basic state. The mode of amplitude $N$ has an inertial period and corresponds to a pulsation circular eddy whose radius varies between

$$
R_{\min }^{2}=\frac{2(1-2 N)}{W(1-W)}
$$

and

$$
R_{\max }^{2}=\frac{2(1+2 N)}{W(1-W)}
$$

with maximum and minimum depth of values

$$
H_{0 \max }=1+2 N
$$

and

$$
H_{0 \text { min }}=1-2 N
$$

respectively. The last two modes corresponding to the frequencies given by (28) are steady clockwise rotations of the ellipse at rates $\omega_{-} / 2$ and $\omega_{+} / 2$. In particular the lowfrequency mode (amplitude $P$ ) corresponds to a slow rotation of the elliptical eddy. For a mean eddy radius equal to 4 times the radius of deformation the rate is $8.5^{\circ}$ per day. Figure 3

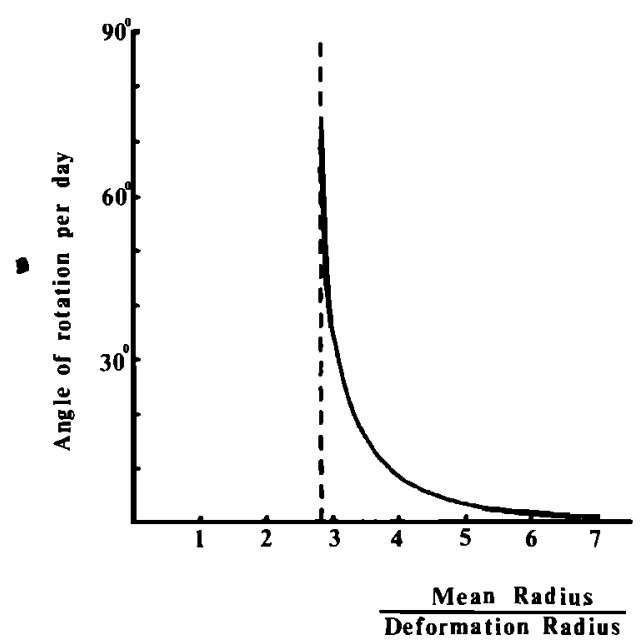

Fig. 3. Plot of the angle of clockwise rotation per day for the low-frequency elliptical mode of a basic circular eddy. Note the rapid drop of rotation rate as the eddy radius increases from its minimum realizable value. The same curve is also valid for eddies of finite eccentricity, provided that the eddy radius is the geometric mean of semimajor and semiminor axes. shows the angle of rotation per day (based on $f=10^{-4} \mathrm{~s}^{-1}$ ) for increasing eddy radius. The sharp decay in rotation rate as the eddy radius increases points to a time scale longer than the inertial period. This is

$$
\text { time scale }=\frac{1}{8 f}\left(\frac{R}{R_{d}}\right)^{4}
$$

The likelihood that this low-frequency mode explains the slow clockwise rotation of elliptical warm-core rings is discussed in section 5 .

\section{Two Exact Nonlinear Solutions}

The previous numerical (subsection $2 c$ ) and asymptotic (section 3) solutions suggest that clockwise rotation and pulsation are two intrinsic modes that a lenslike eddy can undergo, and one wonders whether solutions to the nonlinear equations that exhibit these tendencies can also be constructed. The purpose of this section is to present two such solutions: one corresponds to a steady clockwise rotation of an unchanging elliptical eddy, while the other corresponds to a pulsating circular eddy. These solutions cannot be superimposed in the nonlinear context, but they are new exact analytical solutions to the nonlinear shallow-water equations. In the numerical experiment exposed in subsection $2 d$ these modes of oscillations are present and interacting.

\section{4a. The Rodon: A Clockwise Rotating, Elliptical} Anticyclonic Vortex of Fixed Shape

The solution of this case is

$$
\begin{array}{r}
u=-\Omega y+W(x \cos \omega t-y \sin \omega t) \\
v=\Omega x-W(x \sin \omega t+y \cos \omega t) \\
h=H-\left(A_{0}+A_{1} \sin \omega t\right) x^{2}-2\left(A_{1} \cos \omega t\right) x y \\
-\left(A_{0}-A_{1} \sin \omega t\right) y^{2}
\end{array}
$$

A closed (elliptical) outcropping line requires $A_{0}>0$ and $A_{0}{ }^{2}>A_{1}{ }^{2}$. The edge is a circle for $A_{1}=0$, and the ellipse becomes infinitely long as $A_{1}{ }^{2}$ approaches $A_{0}{ }^{2}$. The six constants $\omega, \Omega, W, H, A_{0}$, and $A_{1}$ are not independent. Substitution in the governing equations yields

$$
\begin{gathered}
W^{2}=\Omega(1+\Omega)+2 A_{0} \\
(1-\omega) W=2 A_{1} \\
2 W A_{0}+(\omega+2 \Omega) A_{1}=0
\end{gathered}
$$

There are thus only three independent parameters, one of which must be $H$, the maximum depth at the eddy center. ( $H$ can be set equal to 1 if the scaling depth is chosen as that center depth). The other two can be chosen to be $A_{0}$ and $A_{1}$, i.e., the parameters that provide the eddy size and ellipticity. These two quantities can be readily evaluated from a satellite 

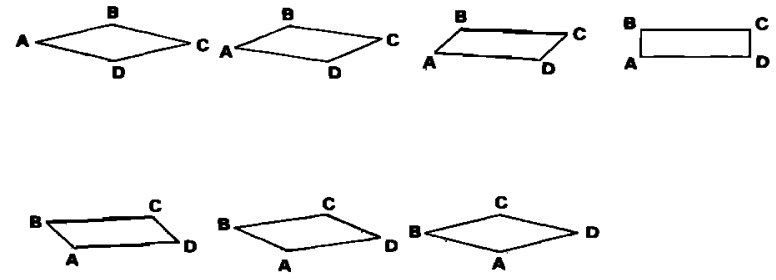

Fig. 4. Example of the time sequence of the relative positions between four neighbor particles inside a steadily rotating elliptical eddy. The time interval between two plots is $1 / 24$ of a period, and the sequence displays a quarter of a period only. During a full period, each particle becomes quite close to all others. The presence of such oscillatory deformation field could greatly homogenize the eddy if mixing were present.

picture. Solving $(32 a-c)$ for $\omega, \Omega$, and $W$, one obtains

$$
\begin{gathered}
\omega=1-\frac{1}{2} \varepsilon_{1}\left[(1+\delta)^{1 / 2}+\varepsilon_{2}(1-\delta)^{1 / 2}\right] \\
\Omega=-\frac{1}{2}+\frac{1}{4} \varepsilon_{1}\left(1-8 \frac{A_{0}}{\delta}\right)(1+\delta)^{1 / 2} \\
+\frac{1}{4} \varepsilon_{1} \varepsilon_{2}\left(1+8 \frac{A_{0}}{\delta}\right)(1-\delta)^{1 / 2} \\
W=2 \varepsilon_{1} \frac{A_{1}}{\delta}\left[(1+\delta)^{1 / 2}-\varepsilon_{2}(1-\delta)^{1 / 2}\right]
\end{gathered}
$$

where the new quantity $\delta$ is defined by

$$
\delta=8\left(A_{0}^{2}-A_{1}^{2}\right)^{1 / 2}
$$

This quantity decreases for increasing eccentricity and for increasing eddy size. The factors $\varepsilon_{1}$ and $\varepsilon_{2}$ are sign flags $( \pm 1)$. There are thus four mathematically possible solutions for given $H, A_{0}$, and $A_{1}$. However, only two are inertially stable (with positive total vorticity $v_{x}-u_{y}+1$ ), and $\varepsilon_{1}=\varepsilon_{2}$. Another requirement of existence is $\delta<1$, which imposes a minimum value on the eddy mean radius (geometric mean of semimajor and semiminor axes)

$$
R_{\min }=8^{1 / 2} R_{d}
$$

where $\boldsymbol{R}_{\mathrm{d}}$ is the radius of deformation based on the center depth. This is identical to the minimum radius discussed by Nof [1981] and Killworth [1983].

Solution $(31 a-c)$ corresponds to an elliptical eddy that rotates steadily clockwise at the angular rate $\omega / 2$. In the rotating coordinate system of the eddy the solution takes the form

$$
\begin{gathered}
u^{\prime}=-\left(\Omega+\frac{\omega}{2}+W\right) y^{\prime} \\
v^{\prime}=\left(\Omega+\frac{\omega}{2}-W\right) x^{\prime} \\
h^{\prime}=H-\left(A_{0}+A_{1}\right) x^{\prime 2}-\left(A_{0}-A_{1}\right) y^{\prime 2}
\end{gathered}
$$

For $\delta$ of order unity, $\omega$ is also of order unity, and the revolution time is on the order of an inertial period. However, for small $\delta$ (wide eddy) the frequency corresponding to $\varepsilon_{1}=$ $\varepsilon_{2}=+1$ is small and on the order of $\delta^{2}$. As for the linearized solution about the circular shape, there is the possibility of a very slow clockwise rotation of the edge, even for moderatesize eddies, probably the one observed for elliptical warm-core rings. In terms of the mean radius (geometric mean of semimajor and semiminor axes), noted $R$, the dimensional rotation rate of the eddy as a whole takes the form

$$
\frac{\omega}{2}=\frac{f}{2}-\frac{f}{4}\left[\left(1+\frac{8 R_{d}{ }^{2}}{R^{2}}\right)^{1 / 2}+\left(1-\frac{8 R_{d}{ }^{2}}{R^{2}}\right)^{1 / 2}\right]
$$

where $R_{\mathrm{d}}$ is the radius of deformation based on the center depth. This expression is identical to the low-frequency root (28) of the linearized theory, and the curve on Figure 3 also applies to eddies of finite eccentricity, provided the radius is taken to be the geometric mean radius. This rotation rate of the eddy as a whole has to be distinguished from the angular rotation of particles, which is generally greater.

The solution has other interesting properties. Since $u_{x}$ $+v_{y}=0$, there is no divergence, and every water column conserves both its vorticity and its depth. More important is the presence of an oscillatory deformation field as seen on Figure 4, which displays the relative positions of four particles as a quarter of period elapses. At the beginning of the sequence, particles $B$ and $D$ are relatively close and, if mixing were present, could easily smooth any difference in their properties. An eighth of a period later, $A$ and $B$ are now close together and can smooth their differences, and so can $C$ and $D$. Another eighth of a period later, $A$ and $D$ are now nearby, and so on. In summary there exists an oscillatory deformation field that maintains communication among particles. Were mixing present, this mechanism could greatly enhance homogenejzation within the eddy. This process is absent in a circular eddy and is stronger for elliptical eddies of great eccentricity.

\section{4b. Pulsation of a Circular Eddy}

The second solution that satisfies the nonlinear problem ((4)-(14) and (16)) exactly corresponds to a pulsating circle. In section 3, such a mode was derived in the case of smallamplitude pulsation. The present solution is valid at finite amplitudes. With $A=C$ and $B=0$ to preserve axisymmetry it follows that $U_{2}=-V_{1}, V_{2}=U_{1}$, and the problem reduces to

$$
\begin{aligned}
\dot{U}_{1} & =-U_{1}{ }^{2}+V_{1}{ }^{2}+V_{1}-2 A \\
\dot{V}_{1} & =-2 U_{1}\left(V_{1}+\frac{1}{2}\right) \\
\dot{A} & =-4 U_{1} A \\
\dot{H}_{0} & =-2 U_{1} H_{0}
\end{aligned}
$$

The system possesses the following first integrals:

$$
\begin{aligned}
U_{1}{ }^{2} & =-(1+2 P)\left(V_{1}+\frac{1}{2}\right)^{2}+Q\left(V_{1}+\frac{1}{2}\right)-\frac{1}{4} \\
A & =-P\left(V_{1}+\frac{1}{2}\right)^{2} \\
H_{0} & =S\left(V_{1}+\frac{1}{2}\right)
\end{aligned}
$$

where $P, Q$, and $S$ are arbitrary constants. Oscillations are possible for $Q^{2} \geq 1+2 P$. Figure 5 displays the phase trajectories in the $U_{1}-V_{1}$ framework. The eddy is widest and shallowest when $U_{1}=0$ and $V_{1}$ is minimum, while it is smallest and deepest when $U_{1}=0$ and $V_{1}$ is maximum. The constants of the first integrals are related to the minima and maxima of radius and center depth by the following expressions:

$$
\begin{gathered}
R_{\max }^{2}=\frac{S}{P}\left[Q+\left(Q^{2}-2 P-1\right)^{1 / 2}\right] \\
R_{\min }{ }^{2}=\frac{S}{P}\left[Q-\left(Q^{2}-2 P-1\right)^{1 / 2}\right] \\
H_{0 \min }=\frac{S}{1+2 P}\left[Q-\left(Q^{2}-2 P-1\right)^{1 / 2}\right] \\
H_{0 \text { max }}=\frac{S}{1+2 P}\left[Q+\left(Q^{2}-2 P-1\right)^{1 / 2}\right] .
\end{gathered}
$$

Time integrations show that the eddy spends more time and evolves more slowly during the wide and shallow phase (small $V_{1}+1 / 2$ and $\left.|A|\right)$ than during the small and deep phase (large 
$V_{1}+1 / 2$ and $\left.|A|\right)$ of the cycle. This asymmetry increases with the pulsation amplitude.

Physically, the oscillations take place as follows. When the eddy is small and deep the azimuthal velocity is too rapid to maintain a geostrophic balance with the sloping interface, and centrifugal forces expand the eddy, which by conservation of mass becomes shallower. A state of equilibrium between azimuthal velocity and interfacial slope is reached and overshot, for the radial velocity is still directly outward. An extreme state of a large and shallow eddy is reached where now the disbalance of geostrophy induces contraction of the eddy. Again, the geostrophic equilibrium is overshot, and the eddy becomes too small and deep. Then the cycle repeats.

\section{Application to Gulf Stream Rings}

In this section an attempt is made to apply the present model to warm-core rings observed north of the Gulf Stream. Although a detailed quantitative comparison is not possible in view of the model assumptions and simplifications, the theory predicts correctly the overall behavior of elliptical anticyclonic rings.

As already pointed out, the observed ellipticity is probably a result of environmental shear [Nof, 1985]. But regardless of the generation mechanism, the model suggests that when an elliptical ring is placed in a resting ocean it cannot remain still and must rotate clockwise. That is to say, once the ellipticity generation mechanism, such as the shear, is relaxed, the ellipse orientation is no longer fixed, and the ring cannot reach any balanced state unless it starts rotating.

To apply the theory to actual rings, we proceed as follows. First, in view of the observations discussed by Csanady [1979] and Joyce [1984] we choose the following numerical values as being typical for warm-core rings :

$$
\begin{array}{rlrl}
H_{0} & \sim 500 \mathrm{~m} & \frac{\Delta \rho}{\rho} & \sim 1.510^{-3} \\
f \sim 10^{-4} \mathrm{~s}^{-1} & R & \sim 100 \mathrm{~km}
\end{array}
$$

These values give a deformation radius $R_{\mathrm{d}}$, of about $27 \mathrm{~km}$. Second, we note that for the purpose of our applications we can consider either the exact solution for a rotating ellipse with a fixed shape (subsection $4 a$ ) or the asymptotic solution (section 3) because the shape of most rings is nearly circular [see, for example, Evans et al., 1984].

It is now recalled that the rotation rate of the eddy as a whole is a function of a single variable, namely, the ratio of the eddy radius to the deformation radius. The typical numbers listed in (40) provide a ratio of 3.7. It is worth noting that this value is consistent with the internal ring structure discussed by Joyce [1984]. For this ratio the theory (see Figure 3 or equation (39)) predicts a rotation rate of the edge of about $12^{\circ}$ per day. This value is very close to the observed rotation rate of a typical Gulf Stream warm-core ring. For example, ring 82B rotated in April 1982 (Plate 1), when it was clearly elliptical, by about $90^{\circ}$ in 10 days, i.e., at a rate of $9^{\circ}$ per day.

From the same typical values listed in (40) the theory also predicts a particle rotation rate (swirl rate) of about $1.810^{-5}$ $\mathrm{s}^{-1}$, which is very close to $210^{-5} \mathrm{~s}^{-1}$ (or $2 \mathrm{~m} \mathrm{~s}^{-1}$ at $100 \mathrm{~km}$ away from the center), as observed by Joyce [1984]. Now, ignoring the eddy radius and basing the calculations solely on this observed swirl rate, we obtain via the theory a eddy rotation rate of $14^{\circ}$ per day. Although this figure is still on the same order of magnitude, it illustrates the sensitivity of the elliptical rotation rate on eddy parameters as pointed by the theory.

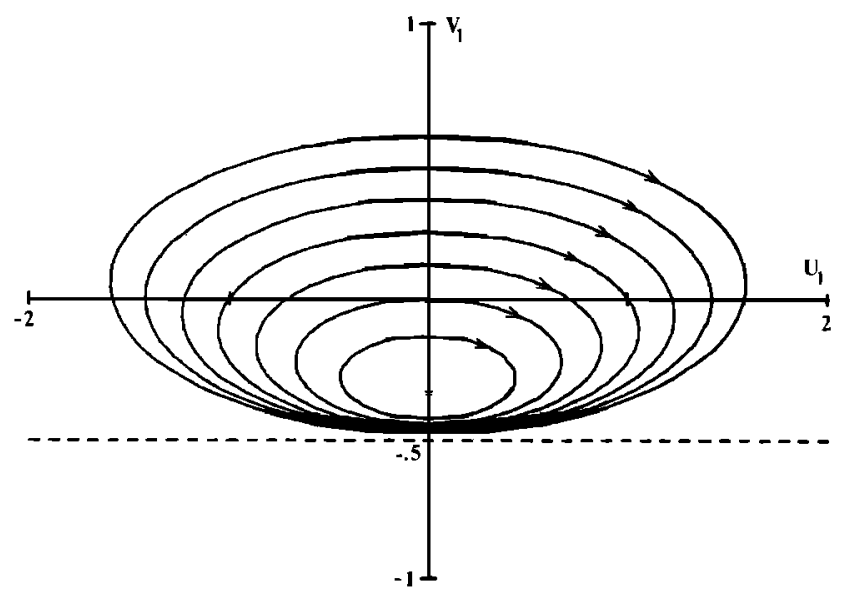

Fig. 5. Phase curves in the $U_{1}-V_{1}$ framework for the nonlinear circular-pulsation solution. The parameter $P$ is set to 4 , and $Q$ varies from curve to curve. Each curve represents one possible pulsation with the eddy widest/shallowest when $V_{1}$ is minimum and smallest/deepest when $V_{1}$ is maximum. The region below the dashed line $\left(V_{1}<-1 / 2\right)$ corresponds to inertially unstable eddies and is not acceptable.

Kirwan et al. [1984] inferred properties of a warm-core ring in the western portion of the Gulf of Mexico from three drifter trajectories and a kinematic model. The eddy was elongated for most of the 3 months during which data were analyzed. However, this ellipticity is very likely the product of a controlled situation (the eddy was hugging the western boundary for most of the three months) rather than the signature of a free evolution following a nonradially symmetric initial state. In particular the eddy maintained an approximate east-west orientation during the 3-month period, with very slow rotation $\left(1^{\circ}\right.$ or $2^{\circ}$ per day) alternatively clockwise and counterclockwise. The present theory applies only to isolated eddies and cannot account for these observations. Their interpretation probably lies in topographic control or ambiant-shear effect [Nof, 1984].

\section{Conclusions}

The $f$ plane, reduced-gravity system of equations admits exact solutions with height and velocity fields respectively quadratic and linear in space. With the implementation of such particular profiles the spatial structure is removed, and the problem reduces to a set of nonlinear, coupled, ordinary differential equations where time is the only variable. This reduced problem has basic properties. First, since the reducedgravity governing equations can always be scaled such that all coefficients are unity, there is no free parameter, and no bifurcation is expected among regimes. Second, there are a sufficient number of invariants (mass, angular momentum, potential vorticity and energy) to ensure integrability, and no chaotic behavior is expected. Finally, the motion of the center of mass is an inertial circular trajectory that can be easily subtracted from the system. All these properties greatly simplify the analysis.

The system of ordinary differential equations in time is exploited in a deductive approach. First, two possible equilibria are isolated: a steady circular eddy (zero eccentricity) and a rectilinear band of warm water (maximum eccentricity). Then, small elliptical departures from circular equilibrium are investigated, and three modes are identified. One mode is a circular pulsation at the inertial frequency, while the other two correspond to steady clockwise rotation of an unchanging elliptical eddy with either a subinertial or superinertial frequency. In 
particular the subinertial mode has a frequency that decays rapidly with increasing eddy radius. Since observed rotation of elliptical anticyclonic rings is also clockwise and subinertial, this mode may be the plausible mechanism that can explain observations. To test this agreement more thoroughly, the rotating elliptical eddy solution is extended from small to finite eccentricity, and detailed comparisons with observations are made.

Besides the plausible explanation for the observed rotation of elliptical Gulf Stream rings, the above solution offers another characteristic. An oscillatory deformation field is present in the system that ensures active communication between particles, not unlike stirring. With an even moderate mixing, as always present in nature, this mechanism can greatly enhance homogeneization of passive tracers in elliptical eddies. This oscillatory deformation field is nil in circular eddies and increases with eccentricity.

It is appropriate to comment on the validity of our assumption regarding the passive and infinitely deep lower layer. To examine this point, consider an elliptical anticyclonic eddy rotating in an ocean with a finite depth, $H$. Let us suppose that the eddy's central depth is $h$, its length scale is $l$, and $\varepsilon$ is a measure of its departure from a circular structure. When $\varepsilon=0$ (i.e., a purely circular eddy) the system obviously has a solution with a stagnant lower layer, even if the lower layer is finite, because no lower-layer fluid is forced to change its depth as the eddy is rotating. If, on the other hand, $\varepsilon$ is not zero, then the nonsymmetrical portions of the eddy will force some lower-layer water columns to change depth. Since the asymmetrical portions have a depth of order $\varepsilon \hat{h}$, potentialvorticity conservation for the lower layer implies that the associated velocities are

$$
u^{\prime}, v^{\prime} \sim 0\left(f \varepsilon \frac{\hat{h}}{H} l\right)
$$

For our model to be valid these lower-layer velocities should be smaller than the smallest velocities resolved within the eddy, which are $O(\varepsilon f l)$. Consequently, when $(\hat{h} / H) \ll 1$, our assumption of no motion in the lower layer is valid. For Gulf Stream rings, $(\hat{h} / H) \sim 0.15$, the lower-layer, neglected motion is an order of magnitude smaller than the computed speeds, and the assumption is justified.

Actual occurrences of Gulf-Stream rings never show pronounced eccentricity and suggest that elongated eddies may not be stable. An investigation of the stability of the solution presented here would be most helpful to answer this point. In particular it may be found that there exists an eccentricity threshold below which eddies are stable and beyond which eddies are unstable and consequently never observed. A nonquasi-geostrophic, infinitesimal-amplitude stability study could also predict the shape of the most unstable mode and provide insight into the decay (splitting?) of an elongated eddy.

The westward drift induced by the beta effect should also be investigated. At a length scale comparable to the deformation radius as for the present solution, the beta term is a small perturbation. An expansion theory could answer the question of combined clockwise rotation and preferential westward translation. In a reduced-gravity model (no lower-layer motion) with a finite area of fluid in motion, Rossby-wave dispersion is excluded. To study dispersion processes, an expanded theory could include both beta effects and lower-layer motion that extends to infinity.

The solutions presented in this paper cannot, unfortunately, be extended to cold-core, cyclonic rings. Either the upper, moving layer extends to infinity, leaving an open area where the lower layer is in contact with the surface, and quadratic depth profiles in $x$ and $y$ are inappropriate, or the cold-core ring is modeled as a mid-water lens of a three-layer system, generating motion in at least the uppermost layer, and two moving layers have to be resolved.

Finally, it is noted that the analytical solutions described in the present paper can be used to assess the accuracy of nonlinear numerical models.

\section{APPENDIX: THE INVARIANTS}

The inviscid model $(1 a-c)$ conserves volume, angular momentum, potential vorticity, and energy, and it is interesting to see which form these invariant properties take for the particular solution exposed above. First, it is noted that

$$
\begin{aligned}
\Delta & =-4\left(U_{1}+V_{2}\right) \Delta \\
\dot{H}_{0} & =-\left(U_{1}+V_{2}\right) H_{0} \\
\dot{M} & =-3\left(U_{1}+V_{2}\right) M \\
\dot{Z} & =-\left(U_{1}+V_{2}\right) Z \\
\dot{Q} & =-3\left(U_{1}+V_{2}\right) Q
\end{aligned}
$$

where $\Delta=A C-B^{2}, H_{0}$ as above, $M=A\left(U_{2}-1 / 2\right)+B\left(V_{2}\right.$ $\left.-U_{1}\right)-C\left(V_{1}+1 / 2\right), Z=\left(V_{1}-U_{2}+1\right)$, and $Q=-A\left(U_{2}^{2}\right.$ $\left.+V_{2}{ }^{2}\right)+2 B\left(U_{1} U_{2}+V_{1} V_{2}\right)-C\left(U_{1}^{2}+V_{1}^{2}\right)+4 \Delta$. It then follows that appropriate combinations of powers of these quantities are time independent. The four independent combinations related to physical quantities are

$$
\text { volume }=\iint h d x y=\frac{\pi}{2} \Delta^{-1 / 2} H_{0}^{2}
$$

$$
\begin{aligned}
& \text { angular momentum }=\iint\left[(x v-y u)+\frac{1}{2}\left(x^{2}+y^{2}\right)\right] h d x d y \\
& =\frac{\pi}{12} \Delta^{-3 / 2} H_{0}^{3} M
\end{aligned}
$$

where the double integral covers the moving eddy. The second term of the angular momentum represents the contribution of the rotating framework, which, in general, depends on time as the eddy contracts and expands.

The moment of inertia, defined as

$$
I=\iint\left(x^{2}+y^{2}\right) h d x d y=-\frac{\pi}{12} \Delta^{-3 / 2} H_{0}{ }^{3}(A+C)
$$

satisfies [Ball, 1963]

$$
\begin{aligned}
I & =-\frac{\pi}{6} \Delta^{-3 / 2} H_{0}{ }^{3}\left(V_{2} A-V_{1} B-U_{2} B+U_{1} C\right) \\
\ddot{I}+I & =\frac{\pi}{6} \Delta^{-3 / 2} H_{0}^{3}(Q+M) \\
& =4(\text { energy) }+2 \text { (angular momentum) }=\text { constant }
\end{aligned}
$$


As a result the expression

$$
\frac{1}{2} \dot{I}^{2}+\frac{1}{2} I^{2}-\frac{\pi}{6} \Delta^{-3 / 2} H_{0}^{3}(Q+M) I
$$

is an invariant of the problem. This fifth invariant is independent of the previous four ones.

Since there are five invariants, the $8 \times 8$ system (4)-(10) with (16) is integrable, and chaotic behavior cannot occur. The three remaining degrees of freedom correspond to three modes of oscillation or growth/decay. These are identified in the text as pulsation of a circular eddy and steady rotations of an elliptical eddy at either a subinertial or superinertial rates.

Acknowledgments. The authors are deeply indebted to R. Evans and the Warm-Gore Rings Program for the permission to reproduce the Gulf Stream ring satellite photographs and to James J. O'Brien and W. R. Young for comments. This research was supported by the Office of Naval Research and constitutes contribution 215 of the Geophysical Fluid Dynamics Institute at the Florida State University.

\section{REFERENCES}

Ball, F. K., Some general theorems concerning the finite motion of a shallow rotating liquid lying on a paraboloid, J. Fluid Mech., 17, 240-256, 1963.

Ball, F. K., The effect of rotation on the simpler modes of motion of a liquid in an elliptic paraboloid, J. Fluid Mech., 22, 529-545, 1965.

Brown, O. B., D. B. Olson, J. W. Brown, and R. H. Evans, Kinematics of a Gulf Stream warm-core ring, Aust. J. Mar. Freshwater Res., 34, $538-545,1984$.

Csanady, G. T., The birth and death of a warm core ring, J. Geophys. Res., 84, 777-780, 1979.

Evans, R., K. Baker, O. Brown, R. Smith, S. Hooker, D. Olson, and the Warm-Core Rings Program Service Office, Satellite images of warm core ring 82-B, Sea surface temperature and a chronological record of major physical events affecting ring structure, report, 25 pp., 18 plates, Woods Hole Oceanogr. Inst., Woods Hole, Mass., 1984.

Flierl, G. R., A simple model for the structure of warm and cold core rings, J. Geophys. Res., 84, 781-785, 1979.

Griffiths, R. W., and P. F. Linden, The stability of vortices in a rotating, stratified fluid, J. Fluid. Mech., 105, 283-316, 1981.

Griffiths, R. W., P. D. Killworth, and M. E. Stern, Ageostrophic instability of ocean currents, J. Fluid Mech., 117, 343-377, 1982.

Hill, M. J. M., On the motion of fluid part of which is moving rotationally and part irrotationally, Phil. Trans. R. Soc. London, 175, $363-411,1884$

Ikeda, M., Instability and splitting of mesoscale rings using a twolayer quasi-geostrophic model on an $f$-plane, J. Phys. Oceanogr., 11 , 987-998, 1981.
Joyce, T. M., Velocity and hydrographic structure of a Gulf Stream warm-core ring, J. Phys. Oceanogr., 14, 936-947, 1984.

Joyce, T. M., et al., Rapid evolution of a Gull Stream warm-core ring, Nature, 308, 837-840, 1984.

Killworth, P. D., On the motion of isolated lenses on a beta-plane, $J$. Phys. Oceanogr., 13, 368-376, 1983.

Kirchhoff, G., Vorlesungen über Mathematische Physik, vol. 1, 466 pp., Teubner, Leipzig, 1876.

Kirwan, A. D., Jr., W. J. Merrell, Jr., J. K. Lewis, R. E. Whitaker, and R. Legeckis, A model for the analysis of drifter data with an application to a warm-core ring in the Gulf of Mexico. J. Geophys. Res., $89,3425-3438,1984$.

Kitano, K., A Kuroshio anticyclonic eddy, J. Phys. Oceanogr., 4, 670 672, 1974.

Lamb, H., Hydrodynamics, 6th Ed., 738 pp., Dover, New York, 1932.

Love, A. E. H., On the stability of certain vortex motions, Proc. London Math. Soc., Ser. 1, 25, 18-43, 1893.

McCalpin, J. D., Analysis of azimuthal mode dynamics of mesoscale eddies, M.S. thesis, 72 pp., Texas A \& M Univ., College Station, Tex., 1984

Mied, R. P., G. J. Lindemann, and J. M. Bergin, Azimuthal structure of a cyclonic Gulf Stream ring, $J$. Geophys. Res., 88, 2530-2546, 1983.

Nof, D., On the $\beta$-induced movement of isolated baroclinic eddies, $J$. Phys. Oceanogr., 11, 1662-1672, 1981.

Nof, D., On the migration of isolated eddies with application to Gulf Stream rings, J. Mar. Res., 41, 399-425, 1983.

Nof, D., On the ellipticity of isolated anticyclonic eddies, Tellus, $37 \mathrm{~A}$, 77-86, 1985.

Paldor, N., Linear stability and stable modes of geostrophic fronts, Geophys. Astrophys. Fluid Dyn., 24, 299-326, $1983 a$.

Paldor, N., Stability and stable modes of coastal fronts, Geophys. Astrophys. Fluid Dyn., 27, 217-228, $1983 b$.

Saunders, P. M., The instability of a baroclinic vortex, J. Phys. Oceanogr., 3, 61-65, 1973

Spence, T. W., and R. Legeckis, Satellite and hydrographic observations of low-frequency wave motions associated with a cold-core Gulf Stream ring, J. Geophys. Res., 86, 1945-1953, 1981.

Stern, M. E., Geostrophic fronts, bores, breaking and blocking waves, J. Fluid Mech., 99, 687-703, 1980.

Stern, M. E., J. A. Whitehead, and B.-L. Hua, The intrusion of a density current along the coast of a rotating fluid, J. Fluid Mech., $123,237-265,1982$.

B. Cushman-Roisin, Mesoscale Air-Sea Interaction Group, Florida State University, Tallahassee, FL 32306.

W. H. Heil, Department of Mathematics, Florida State University, Tallahassee, FL 32306.

D. Nof, Department of Oceanography, Florida State University, Tallahassee, FL 32306.

(Received November 14, 1984; accepted January 25,1985 . 

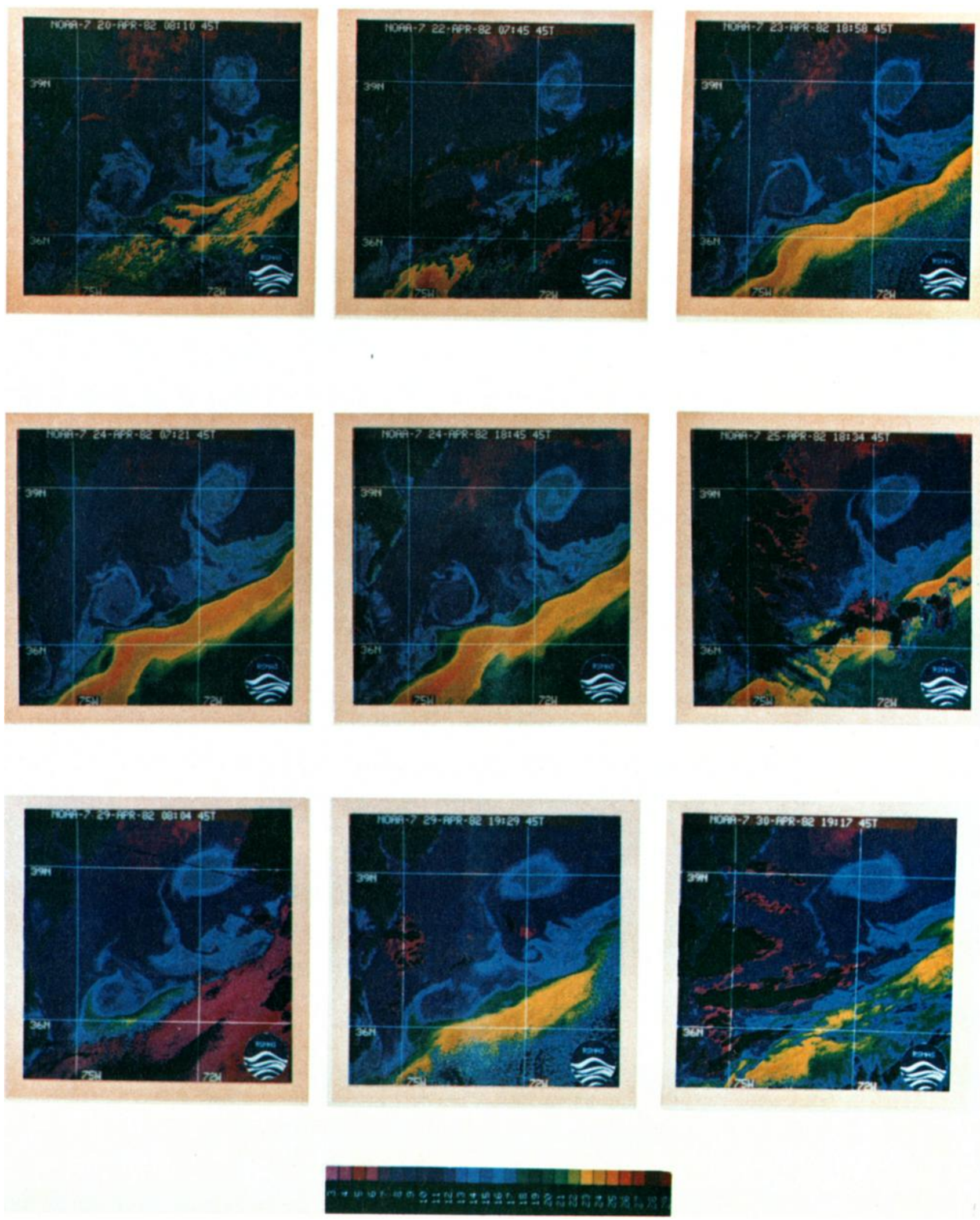

Plate 1. Satellite images of a warm-core ring formed and tracked in 1982 (reproduced with permission from Evans et al. [1984]). The relationship between the actual sea surface temperature and the image color is shown at the bottom. Note that on April 10 (image $a$ ) the elliptical ring was oriented in the north-south direction. Within the next 10 days it rotated slowly in a clockwise manner (images $b-h$ ) until it reached an east-west orientation (image $i$ ). This gives an average rotation rate of $\sim 9^{\circ}$ per day, which is in a qualitative agreement with the rotation rate predicted by the theory $\left(12^{\circ}\right.$ per day). 